\title{
Kinematic error of a harmonic drive
}

\author{
Igor Lyuminarsky ${ }^{1}$, and Stanislav Lyuminarsky ${ }^{1 *}$ \\ ${ }^{1}$ Bauman Moscow State Technical University, 2nd Baumanskaya St., Bldg. 5, Block 1 Moscow, \\ Russian Federation
}

\begin{abstract}
The article deals with the causes for kinematic error of harmonic drives. The error was determined theoretically using a mathematical model of a drive accounting elastic interactions of the drive elements. The paper identifies the main cause for the inherent kinematic error of a harmonic drive featuring a cam wave generator: a variation in a flexible gear deformation at rolling of flexible bearing balls. It was established that the highest kinematic error of a drive is significantly lower than the first harmonic of a flexible gear generating error. There is obtained a cam displacement dependence of the highest kinematic error for VZP-80 drive.
\end{abstract}

\section{Introduction}

Harmonic drives (HD) are widely used in high-precision drive units. Flexible gear deformation allows for multizone, multiple and backlash-free gearing. Such gearing ensures a high kinematic accuracy of harmonic drives. The kinematic accuracy is subject to manufacturing errors and settings of various parts. Their contribution to kinematic error is determined by deformation of the main drive elements. Even in case with perfectly accurate manufacturing of parts harmonic drive has a kinematic error called inherent error [1].

Development of drives featuring enhanced quality characteristics requires using the upto-date design and research methods. Development of improved mathematical models of kinematic error computation for various drives is an essential direction in improvement of performance standards of transmission gears. At design stage, such models will enable to select drive parameters and accuracy class of parts to achieve the required kinematic accuracy.

\section{Problem statement}

The existing methods [2-4] for theoretical calculation of kinematic error (KE) account for deformation of harmonic drive elements and multiple gearing approximately. Flexibility of various units are determined from formulas derived using computational and experimental techniques. At design stage, such approach does not allow predicting KE of harmonic drives to the required accuracy.

\footnotetext{
${ }^{*}$ Corresponding author: katjstas@mail.ru
} 
To predict kinematic error of a harmonic drive it is necessary to calculate elastic interaction between its elements. The mathematical model used herein accounts for: interaction between flank surfaces of teeth, interaction between flexible bearing outer ring (FBOR) and flexible gear, and interaction between balls and FBOR. A resolving system of equations is obtained by using Bubnov-Galerkin method. Nodal flexibilities of flank surfaces of teeth are calculated by the finite element method, nodal flexibilities of a flexible gear and flexible bearing outer ring are calculated by the orthogonal sweep method using the linear theory of shells. The resolving system of equations is solved to find link interaction forces, displacement of links as rigid bodies with relation to the case, turn angles of input and output links the values of which are used to calculate kinematic error of a harmonic drive. The calculation method is described in details in [6].

This article purpose is to identify the main causes of kinematic error of harmonic drives and to evaluate the main components of HD KE using the mathematical model that accounts for flexible interaction between the drive elements.

\section{Inherent kinematic error of a drive}

There may be two causes for inherent kinematic error in a harmonic drive. The first cause is a flexible gear base circle deformation. The base circle deformation results in a pitch point displacement while gears are turning and, thus, in a variation in instant gear ratio causing kinematic error of a drive. The kinematic error component due to this cause is hereinafter denoted as $F^{*}$.

The second cause for inherent kinematic error is a variation in a flexible gear deformation at rolling of flexible bearing balls with relation to the cam main axis. The IKE component due to this cause is hereinafter denoted as $F^{* *}$.

Computational studies of IKE were conducted using the example of VZP-80 harmonic drive featuring a cam wave generator. When determining the first component of IKE, $F^{*}$, there were considered two variants of the drive main parameters. The first variant had even number of teeth $z_{g}=200, z_{b}=202$, the second variant had odd number of teeth $z_{g}=201, z_{b}=203$. Drive pitch modulus is $m=0.4 \mathrm{~mm}$, shifting coefficients are $x_{g}=3,119, z_{b}=3,319$. The computation of $F^{*}$ did not account for the influence on the error from rolling of flexible bearing balls with relation to the cam. For this purpose, in computation, balls were turning together with the cam. The theoretical studies showed that the highest kinematic error is a function of load and varies within: $F_{i o r}^{\prime}=0,01 \ldots 0,03 \mu \mathrm{m}$ for a drive having an even number of teeth; $F_{i o r}^{\prime}=0,001 \ldots 0,006 \mu \mathrm{m}$ for a drive having an odd number of teeth. Small values of $F_{i o r}^{\prime}$ show that the flexible gear base circle deformation may be neglected when determining kinematic error.

When determining the second component of IKE, $F^{* *}$, the influence of the first component, $F^{*}$, was neglected. For this purpose, the elastic interaction of drive elements was calculated for various positions of balls and constant positions of the flexible gear with relation to the cam. The theoretical studies showed that the IKE component resulting from rolling of balls with relation to the cam main axis is a function of load. If torque $M_{C}=10 \ldots 100 \mathrm{HM}$ the highest kinematic error varies within: $F_{i o r}^{\prime}=0,8 \ldots 1,8 \mu \mathrm{m}$ for a drive having an even number of balls; $F_{i o r}^{\prime}=0,05 \ldots 0,4 \mu \mathrm{m}-$ for a drive having an odd number of balls. 


\section{Influence of kinematic error of gears on kinematic error of a drive}

Kinematic error of a gear has two components. The first component of kinematic error results from a gear mandrel displacement with relation to the gear reference axis. This error is taken into account when determining the cam setting errors. The second component of kinematic error of a flexible gear results from kinematic error of the gear cutting machine halving circuit. This KE component is measured by gearing error $F_{c r}$ as per GOST 9178-81 or by accumulative pitch error $F_{p r}$.

This section theoretically studies the influence of kinematic error of a flexible gear on kinematic error of a drive. Rigid gear is assumed to be perfect. The difference between the actual and nominal angular positions of a tooth is determined from the formula:

$$
\Delta \varphi_{i}=2 F_{p r} \cos \left(\varphi_{i}\right) / d_{g}
$$

where $d_{g}, \varphi_{i}$ is pitch circle diameter and angular coordinate of $i$-tooth a flexible gear.

Fig. 1 shows the dependence between VZP-80 kinematic error and the output link turn angle at zero load $\left(M_{C}=0\right)$ and 8 accuracy degree of a flexible gear $\left(F_{p r}=60 \mu \mathrm{m}\right)$. The backlash allowance is $j_{n \text { min }}=1,6 \mu \mathrm{m}$. The figure shows that the kinematic error variation period is equal to the time required for the flexible gear to turn to $180^{\circ}$ with relation to the cam, which corresponds to the HD turn with relation to the case to angle $\varphi_{g}=107^{\prime}$.

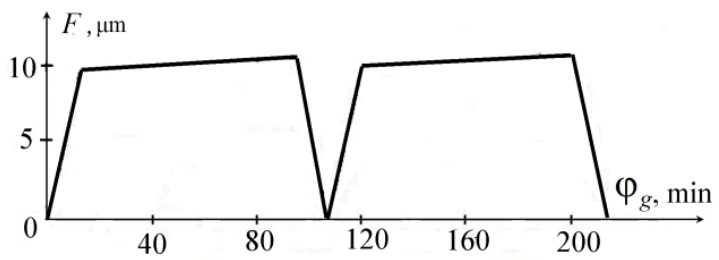

Fig. 1. Kinematic error of VZP-80 at $M_{C}=0$

While the cam is turning, kinematic error $F$ is increases approximately to $10 \mu \mathrm{m}$. Horizontal segments correspond to zero backlash. The highest kinematic error $F_{i o r}^{\prime} \approx 10 \mu \mathrm{m}$.

Let us consider a cause for an insignificant variation of $F$ after the backlash closing. If a tooth in one area of engagement is ahead of the nominal position the respective tooth in the opposite area of engagement is behind the nominal position. In this case the HD kinematic error the occurrence increases the teeth interaction forces along the driving flanks in one area of engagement and along non-driving flanks in another (opposite) area of engagement. As a result, the position of a flexible gear insignificantly changes.

Increase in resistance $M_{C}$ is accompanied with increase in teeth deformation and backslashes between non-driving flanks in both areas of engagement. Therefore, at high loads, increase in the $F_{p r}$ error a flexible gear will not result in disengagement of all the teeth in one of the areas of engagement and teeth contact on non-driving flanks. Increase in interaction forces of teeth in one of the areas of engagement will be compensated by decrease of the same in the opposite area. That is why kinematic error of a drive is significantly lower than kinematic error of a flexible gear. 
Fig. 2 shows the load dependence of the highest kinematic error of a drive for $F_{p r}=60 \mu \mathrm{m}$. The oscillating behaviour of this dependence is associated with a variation in the number of balls in contact with the flexible bearing outer ring.

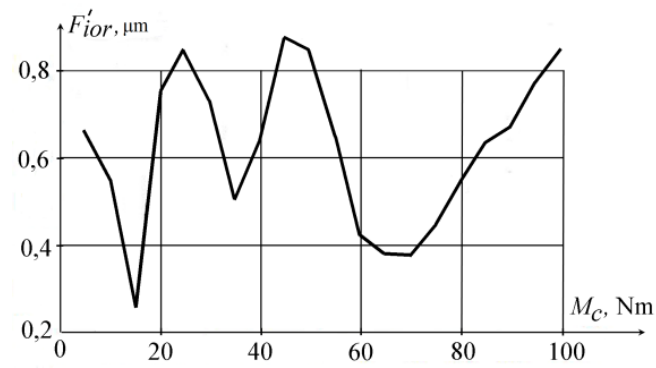

Fig. 2. Highest kinematic error of VZP-80 caused by a flexible gear generating error ( $\left.F_{p r}=60 \mu \mathrm{m}\right)$

The highest kinematic error of a drive, $F_{i o r}^{\prime}$, for $M_{C}=5 \ldots 100 \mathrm{Nm}$ is by $10 \ldots 20$ times lower than at zero load. At these values of torque, $F_{i o r}^{\prime}$ is much lower than accumulative pitch error $F_{p r}$ of a flexible gear.

\section{Influence of a wave generator setting error on kinematic error of a drive}

This section deals with the influence of a wave generator setting error on kinematic error of VZP-80. Errors of parts that influence on the generator setting can be divided into three groups.

1. Static errors. These errors cause a periodic component of kinematic error that varies at a circular frequency of a wave generator $\omega_{h}$.

2. Errors rotating together with a flexible gear. These errors cause a periodic component of kinematic error that varies at circular frequency $\omega_{h}^{(g)}=\omega_{h}(1-1 / u)$.

3. Errors rotating at an angular velocity of a wave generator. These errors do not cause kinematic error.

HD gear ratio is quite high $(u=100)$, so in order to facilitate the calculations we can assume that $\omega_{h}^{(g)} \approx \omega_{h}$. Then, all the errors that influence the cam setting with relation to the case can be summed up.

Fig. 3 shows the $e_{h}$ cam displacement dependence of the highest kinematic error $F_{i o r}^{\prime}$ of VZP-80 for various torques $M_{C}$. The figure shows that the dependence is non-linear. With increase in the cam displacement, at first, $F_{i o r}{ }^{\prime}$ increases insignificantly, and then rises sharply. Such nature of the error variation is due to the fact that at high values of cam displacement $e_{h}$ all teeth of one of two half-waves disengage. 


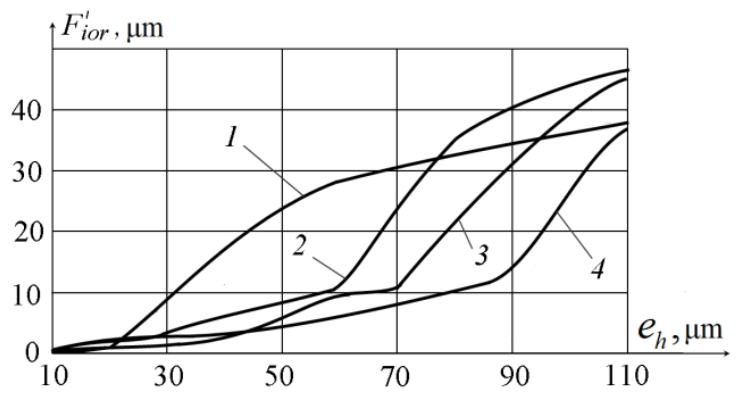

Fig. 3. Highest kinematic error of VZP-80 caused by cam displacement: $1-M_{C}=1 \mathrm{Nm} ; 2-$ $M_{C}=10 \mathrm{Nm} ; 3-M_{C}=50 \mathrm{Nm} ; 4-M_{C}=100 \mathrm{Nm}$

\section{Conclusion}

1. We have identified the causes for inherent kinematic error of harmonic drives. It is established that such error for VZP-80 does not exceed $1.8 \mu \mathrm{m}$

2. The highest kinematic error, $F_{i o r}^{\prime}$, is much lower than accumulative pitch error of a flexible gear. This is due to the fact that teeth in one area of engagement are ahead of the nominal position while teeth in the opposite area of engagement are behind the nominal position.

3. The article considers only the first harmonic component, $F_{p r}$. Even components will have higher influence on $F_{i o r}^{\prime}$ than odd ones because teeth are ahead of the nominal position in both areas of engagement.

4. The cam setting error causes oscillations of harmonic drive elements. These oscillations cause the wave generator dependence of $F_{i o r}^{\prime}$ [4].

\section{References}

1. S.N. Istomin, Kinematic Accuracy of Instrument Harmonic Drives (M.: Machine Engineering, 1987)

2 G.A. Timofeev, Yu.V. Kostikov, Degree of Harmonic Drive Manufacturing Errors Impact on Kinematic Accuracy, Machine Drives and Parts, no. 3(20), pp. 10-13 (2016)

3. Yu.V. Kostikov, G.A. Timofeev, F.I. Fursyak, Kinematic Error and Backlash of External Deformation Harmonic Drives, Proceedings of Higher Educational Institutions. Machine Building, no. 8, pp. 30-34 (2013)

4. P.K. Popov, L.O. Shtripling, Dynamic Models of Kinematic Error Occurrence in a Harmonic Drive, Proceedings of Higher Educational Institutions. Machine Building, no. 1, pp.46-50 (1986)

5. I.E. Lyuminarsky, S.E. Lyuminarsky, Calculation of Kinematic Error of a Harmonic Drive as an Elastic One-Side Contact System, Proceedings of Higher Educational Institutions. Machine Building, no. 8, pp. 9-19 (2008) 